\title{
Bevacizumab combined with pemetrexed successfully treated lung adenocarcinoma complicated with pulmonary tumor thrombotic microangiopathy: a case report and literature review
}

\author{
Lan $\mathrm{Lu}^{1}$, Zhijie Wang ${ }^{2}$, Huawei $\mathrm{Li}^{1}, \mathrm{Xue} \mathrm{Li}^{1}$, Shuangyue $\mathrm{Ma}^{1}$, Luhua Wang ${ }^{3}$, Boyan Yang ${ }^{1,4}$ \\ ${ }^{1}$ Department of Comprehensive Oncology, National Cancer Center/National Clinical Research Center for Cancer/Cancer Hospital \& Shenzhen \\ Hospital, Chinese Academy of Medical Sciences and Peking Union Medical College, Shenzhen, China; ${ }^{2}$ Department of Medical Oncology, State \\ Key Laboratory of Molecular Oncology, National Cancer Center/National Clinical Research Center for Cancer/Cancer Hospital, Chinese Academy \\ of Medical Sciences and Peking Union Medical College, Beijing, China; ${ }^{3}$ Department of Radiation Oncology, National Cancer Center/National \\ Clinical Research Center for Cancer/Cancer Hospital \& Shenzhen Hospital, Chinese Academy of Medical Sciences and Peking Union Medical \\ College, Shenzhen, China; ${ }^{4}$ Department of Comprehensive Oncology, National Cancer Center/National Clinical Research Center for Cancer/ \\ Cancer Hospital, Chinese Academy of Medical Sciences and Peking Union Medical College, Beijing, China \\ Correspondence to: Dr. Boyan Yang. Department of Comprehensive Oncology, National Cancer Center/National Clinical Research Center for \\ Cancer/Cancer Hospital \& Shenzhen Hospital, Chinese Academy of Medical Sciences and Peking Union Medical College, Shenzhen 518116, \\ China; Department of Comprehensive Oncology, National Cancer Center/National Clinical Research Center for Cancer/Cancer Hospital, Chinese \\ Academy of Medical Sciences and Peking Union Medical College, Beijing 100021, China. Email: yby6599@aliyun.com.
}

\begin{abstract}
Pulmonary tumor thrombotic microangiopathy (PTTM) is a rare but severe pulmonary complication of malignant tumors. It is characterized by the presence of multiple microscopic tumor emboli attached to the endothelium of small pulmonary arteries and induces fibrointimal proliferation and the activation of coagulation. The main clinical manifestations of PTTM are dyspnea, dry cough, hypoxemia, pulmonary hypertension, right heart failure, thrombocytopenia, and disseminated intravascular coagulation (DIC). Chest computed tomography (CT) shows no distinctive findings, and PTTM is often unrecognized and universally underdiagnosed, with an appalling prognosis. An antemortem diagnosis of PTTM is also difficult due to a lack of specific clinical and imaging features. Moreover, there is presently no therapeutic management and the illness rapidly progresses to death. Early identification and timely and effective use of oncotherapy can help to alleviate symptoms and improve prognosis. According to recent reports, targeting angiogenesis factors including platelet-derived growth factor (PDGF) and vascular endothelial growth factor (VEGF) have marginally improved survival. In this article, we report the case of a patient with advanced lung adenocarcinoma complicated with PTTM and DIC. A combined therapeutic regimen of bevacizumab with pemetrexed successfully improved chest CT findings, respiratory symptoms, DIC, and short-term outcomes. Anti-angiogenesis drugs modify the pulmonary vascular structure, and rapidly improve the lung tumor microenvironment. And therefore, it may be a potentially effective drug for the treatment of PTTM.
\end{abstract}

Keywords: Pulmonary tumor thrombotic microangiopathy (PTTM); lung adenocarcinoma; disseminated intravascular coagulation (DIC); bevacizumab; chemotherapy

Submitted Sep 24, 2020. Accepted for publication Dec 26, 2020.

doi: 10.21037/apm-20-2220

View this article at: http://dx.doi.org/10.21037/apm-20-2220

\section{Introduction}

Pulmonary tumor thrombotic microangiopathy (PTTM) is a rare and severe pulmonary complication of malignant tumors, and was first proposed by von Herbay in 1990 (1). The pathogenesis of PTTM is still unclear, yet its main pathological characteristics include the presence of non- 
occlusive microtumor emboli in pulmonary arterioles, the activation of coagulation, diffuse thrombotic microvascular emboli, intimal fibroblast proliferation in small pulmonary vessels, luminal stenosis, and remodeling of pulmonary vascular structure (1). PTTM can occur at any stage of any tumor or before tumor diagnosis, which is usually found in poorly differentiated adenocarcinomas (most commonly in gastric adenocarcinoma, followed by lung adenocarcinoma, breast cancer, and ovarian cancer). The clinical manifestations of PTTM lack specificity and mostly appear as progressive dyspnea, dry cough, hypoxemia, respiratory failure, right heart failure, anemia, thrombocytopenia, elevated D-dimer, and pulmonary hypertension, with no evidence of pulmonary embolism. Chest computed tomography (CT) exhibits no distinctive features from other lung diseases. Furthermore, there is no currently therapeutic management of PTTM; the prognosis is poor and the mortality rate is remarkably high ( $>90 \%)$, with the illness rapidly progressing to death. Moreover, an antemortem diagnosis of PTTM is difficult due to the lack of specific clinical and imaging features, and thus, PTTM is largely unrecognized and universally underdiagnosed. According to recent reports, targeting angiogenesis factors including platelet-derived growth factor (PDGF) and vascular endothelial growth factor (VEGF) can help to alleviate symptoms and marginally improve survival (2).

This article reports a case of advanced lung adenocarcinoma complicated with PTTM and disseminated intravascular coagulation (DIC). Clinical diagnosis of PTTM was made after discussion and recognition, followed by a treatment regimen of bevacizumab with pemetrexed. The patient's condition was rapidly relieved despite the short remission time. Herein, we present our clinical knowledge and experience, review recent literature reports regarding PTTM, and examine the clinical features, pathophysiology, clinical detection and diagnosis, and therapeutic strategies with the aim of improving awareness of clinical diagnosis and developing better therapeutic options.

We present the following article in accordance with the CARE reporting checklist (available at http://dx.doi. org/10.21037/apm-20-2220).

\section{Case presentation}

\section{Medical history}

All procedures performed in studies involving human participants were in accordance with the ethical standards of the institutional and/or national research committee(s) and with the Helsinki Declaration (as revised in 2013). Written informed consent was obtained from the patient.

Two years ago, a 51-year-old male patient with no history of chronic obstructive pulmonary disease (COPD), heart disease, or hypertension was diagnosed with lung adenocarcinoma of the right upper lobe (cT1N3M1 stage IVB). The patient also had lung, right adrenal, and multiple bone metastases. He underwent six cycles of chemotherapy with pemetrexed plus cisplatin, with only a partial response (PR). Upon followup, genetic testing showed endothelial growth factor receptor (EGFR) p.L858R Exon21 mutation and amplification. The patient subsequently underwent gefitinib combined with bevacitumab resulting in a progression-free survival (PFS) of 11 months. Re-examination results discovered brain metastases; the patient then underwent treatment with osimertinib combined with bevacitumab for 6 months, with a stable disease (SD) treatment response. During this period, the right adrenal metastasis was treated with radiotherapy. There was also a pulmonary interstitial lesion.

Next, the treatment regime was adjusted to albumin paclitaxel, nedaplatin, and bevacitumab for five cycles. The patient subsequently suffered liver metastasis and pleural effusion. Genetic testing of the pleural fluid showed the following: EGFR p.L858R Exon21 and MET protooncogene (MET) mutation and amplification; positive programmed death-ligand 1 (PD-L1) [tumor proportion score $(\mathrm{TPS})=50 \%]$; cyclin D1(CCND1)/fibroblast growth factor 3 (FGF3)/fibroblast growth factor 4 (FGF4)/ fibroblast growth factor 19 (FGF19) amplification; and tumor protein 53 (TP53) mutation. The therapeutic regime was adjusted to dacomitinib combined with crizotinib resulting in a PFS of 4 months. The results of reexamination revealed pulmonary lesion progression and interstitial lung lesions, and dacomitinib and crizotinib were ceased. The patient then received chemotherapy with pemetrexed plus cisplatin, however he did not complete the cisplatin treatment due to obvious fatigue. His family history was unremarkable. He was a former tobacco smoker with 30 pack-years of smoking history.

\section{Clinical manifestations and laboratory tests}

Following admission, the patient developed progressively aggravated dyspnea, palpitations, coughing with little sputum, blood-stained sputum, severe fatigue, listlessness, and clear consciousness. This was accompanied by an Eastern Cooperative Oncology Group (ECOG) score of 3 , heart rate 130 beats/min, and breathing 30 times/min. 

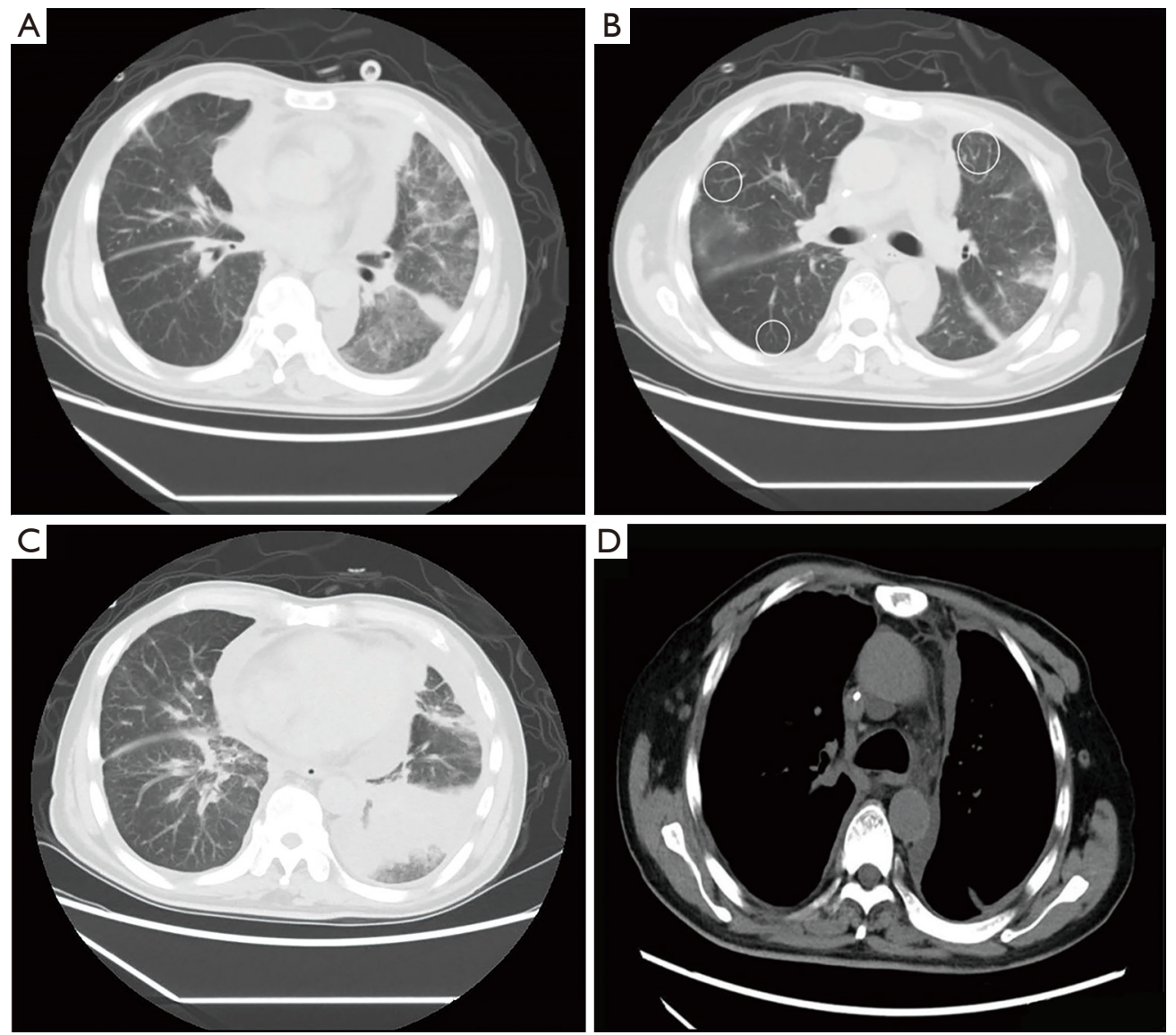

Figure 1 Chest CT images showing: (A,B,C) multiple patchy, ground glass, and nodule-like shadows in both lungs of the lung window; (B, white circles) "tree-in-bud" pattern in both lungs; (D) diffuse and irregular thickening in the bilateral pleura, pleural metastasis.

He also exhibited semi-reclining position (not lying flat), multiple subcutaneous ecchymoses, audible dry and wet rales in both lungs (mainly in the left lower lung), and severe pitting edema of both lower limbs. Moreover, his platelets were progressively decreased, metastatic tumor cells were found by bone marrow examination, there were eight megakaryocytes, and occasional platelets in the whole film. Candida albicans was found in the sputum culture, and Staphylococcus aureus was found in the hydrothorax culture.

Furthermore, the patient's white blood cell count was $9.43 \times 10^{9} / \mathrm{L}$ and platelet count was $8 \times 10^{9} / \mathrm{L}$. Blood gas analysis ( $5 \mathrm{~L}$ oxygen by nasal cannula) showed that his blood oxygen saturation $\left(\mathrm{SpO}_{2}\right)$ was $94.7 \%$, partial pressure of oxygen $\left(\mathrm{PO}_{2}\right)$ was $68 \mathrm{mmHg}$, partial pressure of carbon dioxide $\left(\mathrm{PCO}_{2}\right)$ was $35.9 \mathrm{mmHg}$, and hyoxemia. Also, Coagulation mechanism: prothrombin time and international normalized ratio (INR) were prolonged, fibrinogen was reduced to $0.72 \mathrm{~g} / \mathrm{L}, \mathrm{D}$-dimer reached the maximum clinical laboratory limit $(>20.00 \mu \mathrm{g} / \mathrm{mL})$, fibrin degradation product (FDP) was $87.81 \mu \mathrm{g} / \mathrm{mL}$, antithrombin III was decreased to $53 \%$, and B-type natriuretic peptide precursor (Pro-BNP) was $798.50 \mathrm{pg} / \mathrm{mL}$. Moreover, the three aspects of myocardial infarction were remarkably elevated; high-sensitivity T (cTnI) was at $33.94 \mathrm{pg} / \mathrm{mL}$, and lactate dehydrogenase (LDH) was at $988 \mathrm{U} / \mathrm{L}$.

Note: the patient's Chinese DIC Scoring System (CDSS) score was 10 (3), and his International society on Thrombosis and Hemostasis (ISTH) score was 7 (4).

\section{Imaging examination}

Chest CT (Figure 1) revealed the following: (I) the primary lung cancer in the posterior segment of the right upper lobe was slightly larger than before. (II) Multiple patchy and nodule-like shadows were found in both lungs (primarily 
Table 1 Dynamic changes of blood routine

\begin{tabular}{lcccc}
\hline Date & WBC $\left(\times 10^{9} / \mathrm{L}\right)$ & $\mathrm{RBC}\left(\times 10^{12} / \mathrm{L}\right)$ & $\mathrm{Hb}(\mathrm{g} / \mathrm{L})$ & $\mathrm{PLT}\left(\times 10^{9} / \mathrm{L}\right)$ \\
\hline 23-Mar & 10.74 & 3.86 & 131 & 55 \\
24-Mar & 10.77 & 3.73 & 122 & 15 \\
27-Mar & 9.43 & 3.62 & 118 & 8 \\
28-Mar & 7.34 & 3.05 & 101 & 41 \\
10-Apr & 5.85 & 2.43 & 78 & 56 \\
\hline
\end{tabular}

March 27, anti-tumor therapy. WBC, leukocyte; RBC, erythrocyte; $\mathrm{Hb}$, haemoglobin; PLT, platelet.

Table 2 Dynamic changes of D-dimer, FDP, LDH, and Pro-BNP

\begin{tabular}{lcccc}
\hline Date & D-Dimer $(\mu \mathrm{g} / \mathrm{mL})$ & FDP $(\mu \mathrm{g} / \mathrm{mL})$ & LDH $(\mathrm{U} / \mathrm{L})$ & $\begin{array}{c}\text { Pro-BNP } \\
(\mathrm{pg} / \mathrm{mL})\end{array}$ \\
\hline 23-Mar & $>20.00$ & 87.81 & 916 & 583 \\
27-Mar & 20.00 & 56.4 & 988 & 2536 \\
6-Apr & 0.94 & 1.72 & 372 & 112.7 \\
\hline
\end{tabular}

FDP, fibrinogen degradation products; LDH, lactate dehydrogenase; pro-BNP, pro brain natriuretic peptide.

in the upper lobe of the left lung) with a significantly larger range than before. There was diffuse inhomogeneous reticular interstitium thickening in both lungs, which partly involved the bronchi in the lower lobe of the left lung and made them narrow and obstructed. The scope of obstructive consolidation shadows in the lower lobe of the left lung was significantly larger than before. (III) There was diffuse and irregular thickening of the bilateral pleura, which was considered to be more serious pleural metastasis than before. There was also more bilateral pleural effusion than there was previously.

Echocardiography (ECG) showed that the left ventricular ejection fraction was decreased from $80 \%$ to $52 \%$, with a small amount of pericardial effusion. ECG also revealed sinus tachycardia, rapid paroxysmal atrial fibrillation, high right ventricular voltage, incomplete right bundle branch block, an abnormal T-wave, and right-axis deviation.

\section{Clinical diagnosis}

The patient was diagnosed with the following: (I) adenocarcinoma of the right upper lobe with multiple metastases of both lungs, liver, brain, right adrenal gland, pleura, bone, and bone marrow invasion. He also had multiple metastases of the supraclavicular, mediastinum, and bilateral hilar lymph node (LN) (cT1N3M1c stage IVB), EGFR p.L858R Exon21, and MET mutations, as well as progression after targeted comprehensive therapy; (II) PTTM; (III) DIC; (IV) hyoxemia; (V) severe thrombocytopenia; (VI) Interstitial lung disease (ILD); (VII) Pulmonary lymphangitic carcinomatosis (PLC); (VIII) severe infection of both lungs (bacterial and fungal); (IX) bilateral malignant pleural effusion; (X) severe protein energy malnutrition; (XI) myocardial damage and cardiac insufficiency grade III (right heart failure); (XII) rapid arrhythmia-paroxysmal atrial fibrillation and right bundle branch block.

\section{Treatment and clinical outcomes}

The patient's condition did not improve following treatment of infection, asthma, pleural drainage, methylprednisolone, thrombopoietin (TPO), platelet transfusion, low molecular heparin, fresh-frozen plasma to correct DIC, and nutritional support to correct low protein. The patient and his family members had a strong desire for treatment. After adequate communication, pemetrexed disodium $500 \mathrm{mg} / \mathrm{kg}$, D1, and bevacizumab $5 \mathrm{mg} / \mathrm{kg}$, D1, were used. Following treating with antineoplastons, his symptoms and signs improved rapidly, and his DIC and heart failure were corrected. On the second day, his dyspnea was improved, and he could complete walking exercises. His cough was gradually relieved, without any further hemoptysis, together with significantly improved fatigue, sleep, food intake, and mental and physical status. The patient's heart rate was 90 beats/min, breathing was 22 breaths/min, and $\mathrm{SpO}_{2}$ was $99-100 \%$. No fresh ecchymoses were found in the skin, no dry or wet rales were heard in both lungs, and the patient's severe pitting edema of both lower limbs had disappeared.

According to the laboratory examinations, DIC indicators and imaging were all improved. Cancer Antigen 125 (CA125), cytokeratin 19 fragment CYFRA21-1, carcinoembryonic antigen (CEA), and neuron-specific enolase (NSE) were slightly increased. Hemoglobin was decreased from 118 to $78 \mathrm{~g} / \mathrm{L}$. Platelets were at $56 \times 10^{9} / \mathrm{L}$, fibrinogen was at $1.14 \mathrm{~g} / \mathrm{L}, \mathrm{D}$-dimer was at $0.94 \mu \mathrm{g} / \mathrm{mL}$, fibrin degradation products (FDP) were at $1.72 \mu \mathrm{g} / \mathrm{mL}$, pro$\mathrm{BNP}$ was at $112.70 \mathrm{pg} / \mathrm{mL}, \mathrm{cTnI}$ was at $29.60 \mathrm{pg} / \mathrm{mL}, \mathrm{LDH}$ was at $372 \mathrm{U} / \mathrm{L}$, C-reactive protein (CRP) was at $5.62 \mathrm{mg} / \mathrm{L}$, and procalcitonin was at $0.317 \mathrm{ng} / \mathrm{mL}$.

Note: the patient's CDSS score was 3 . The dynamic changes in blood routine are shown in Table 1. The dynamic changes of D-dimer, FDP, LDH, and Pro-BN are shown in Table 2. 

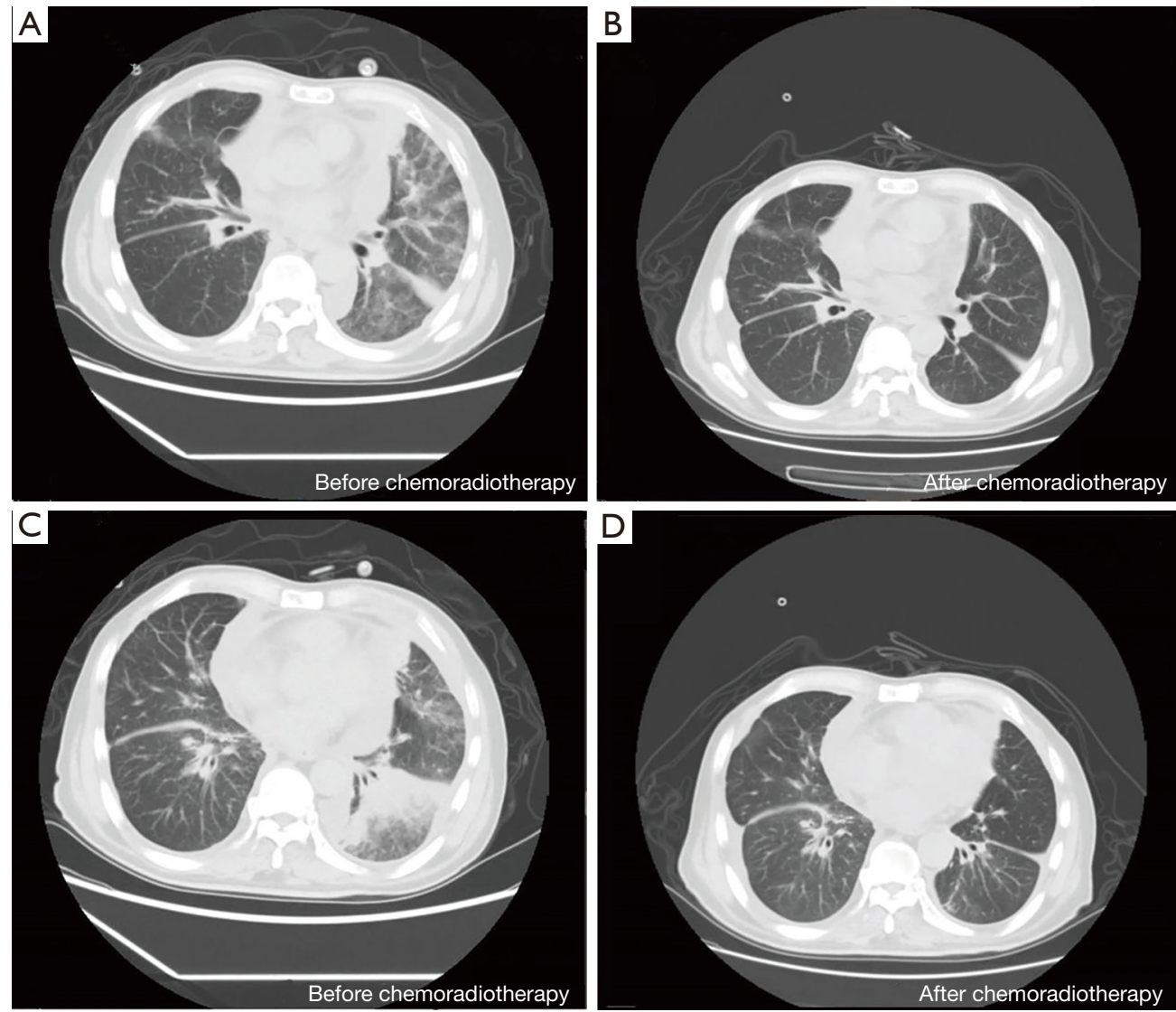

Figure 2 Chest CT imaging showing multiple patchy, nodule-like shadows and diffused inhomogeneous reticular interstitium thickening in both lungs, which were remarkably smaller than pre-chemotherapy.

Chest CT results (Figure 2) showed the following: (I) the primary lung cancer in the posterior segment of the right upper lobe was similar to before. (II) Multiple patchy and nodule-like shadows in both lungs were remarkably smaller than they were previously. (III) Diffused inhomogeneous reticular interstitium was thickened in both lungs, which partly involved the bronchi in the lower lobe of the left lung and made them narrow and obstructed; obstructive consolidation was seen in the lower lobe of the left lung to a lesser extent than before.

After 19 days of stable illness, he relapsed with progressive dyspnea, cough, fatigue, and was extremely dejected. The patient and his family members abandoned treatment and discharged immediately. The patient died after 2 days, and we were informed by telephone follow-up.

\section{Discussion}

In 1990, von Herbay et al. (1) first reported and proposed the concept of PTTM. He observed three patients with dyspnea and unexplained pulmonary hypertension over a 4-year period, and all of them died of right heart failure within a short period of time. Autopsy of these patients found tumors with concurrent metastases and tumorrelated microvascular diseases that led to fatal pulmonary hypertension, which were different from the vascular emboli of pulmonary tumors and tumor emboli of primary lung cancer. Subsequently, of 630 tumor autopsies, 21 cases were determined to be consistent with the clinical manifestations of PTTM. As shown by pathological examination, tumor cells, which were single free, small clumps or attached to the vascular endothelium, were found in the lumen of pulmonary arterioles, and there were thrombotic substances around them. Tumor cell adhesion to the endothelium causes fibrointima eccentric hyperplasia and progressive stenosis of the vascular lumen, but will not lead to lumen occlusion. Immunoreactive tumor cells were detected on blood vessel walls and periarterial lymphatic vessels. Among 
the 21 PTTM cases, 18 were accompanied by cancerous lymphangitis, although it remains unclear whether there is an intrinsic relationship between PTTM and cancerous lymphangitis. Furthermore, 10 cases were accompanied by pulmonary hypertension. Thromboembolism in PTTM is considered to be the main cause of increased pulmonary vascular resistance, despite negative imaging in some cases. In contrast to other pulmonary thromboembolic diseases, PTTM exhibits a significant intimal hyperplasia in pulmonary arterioles.

PTTM is a serious condition, and will rapidly progress to respiratory failure, right heart failure, DIC, and ultimately death following the onset of clinical symptoms. The antemortem diagnosis rate of PTTM is low. Owing to the inability of patients to tolerate invasive pathological biopsy and insufficient attention from clinicians, the diagnosis is often easily missed or is misdiagnosed. The autopsy detection rate is between $1.4 \%$ and $3.3 \%$ (5). Crucially, early diagnosis of PTTM is a prerequisite for treatment (6).

\section{Features of clinical and laboratory examinations}

The most common pathological type of PTTM is poorly differentiated adenocarcinoma (most commonly in gastric adenocarcinoma, followed by lung adenocarcinoma) (1), together with other common tumors with low proportion, such as breast, ovarian, and bladder cancers. Gainza et al. (7) believed that micro-tumor metastasis not only occurred in lung, but also in other organs, leading to multi-organ tumor thrombotic microangiopathies, with PTTM representing only part of these diseases. PTTMs are mostly characterized by rapid and progressively aggravated dyspnea followed by a dry cough, chest pain, and hemoptysis. Recent studies have shown that dry cough in PTTM patients may originate from airway hyperresponsiveness induced by tumor cellsecreted cytokines, and thus, dry cough may be a valuable diagnostic criterion (8). Pulmonary hypertension and right heart failure are frequently accompanied by symptoms including edema of both lower limbs, nausea, vomiting, and loss of appetite. However, it has been reported that only $15-20 \%$ of pulmonary hypertension can induce typical clinical manifestations of right heart failure.

As has been previously reported, more than half of PTTM cases complicated with DIC are accompanied by thrombocytopenia, elevated D-dimer, and $\operatorname{FDP}(5,9)$, which indicates that the coagulation system is activated. Gainza et al. (7) analyzed three cases of PTTM and found characteristics similar to laboratory examinations, including thrombocytopenia, hemolysis, aberrantly elevated serum LDH, and abnormal blood coagulation. Broken red blood cells and decreased platelet count in PTTM patients are considered to represent the mechanical destruction of red blood cells and platelet aggregation caused by blood flowing through narrow lung capillaries. Arterial blood gas analysis often suggests hypoxemia and even type I respiratory failure.

In this study, the patient exhibited clinical characteristics such as progressive dyspnea and hypoxemia, accompanied by DIC manifestations (including increased D-dimer, FDP, and obvious thrombocytopenia), as well as right heart failure manifestations (such as anemia, significantly increased LDH, edema of both lower limbs, and elevated blood pressure in a short period of time). All of these symptoms were consistent with the clinical manifestations of PTTM.

\section{Features of imaging and ultrasound}

The chest imaging examination of PTTM lacks specificity, and ordinary chest CT may show no obvious abnormalities. Meanwhile, high-resolution CT (HRCT) of the chest may identify abnormal changes in the lungs, and could thereby reduce the rate of missed diagnoses. Positron emission tomography-CT (PET-CT) may show high uptake at the primary tumor site and at the metastatic tumor embolism site in the lungs.

Chest HRCT features of PTTM include the following: (I) extensive reticular interstitial changes (thickened lung lobular septum) $(10,11)$; (II) diffused ground glass opacity (GGO) in both lungs $(10,11)$; (III) diffused small nodules in the central lobules of both lungs, forming a "tree-in-bud" pattern with surrounding blood vessels (12-14); and (IV) small scattered pieces of consolidation under bilateral pleura. Katayama et al. (10) reported that GGO represents diffused alveolar injury instead of bleeding due to inflammatory mediators. Gorospe Sarasúa et al. (14) considered that among the four CT manifestations of PTTM, the "tree-in-bud" pattern is the only imaging indicator that can reflect vascular factors, although it should be differentiated from infectious bronchitis. Kayatani et al. (15) suggested that the scattered fine granular shadows in both lungs might be an early imaging feature of PTTM. Toyonaga et al. (16) dynamically observed the imaging evolution of PTTM, and found that chest HRCT was normal in the early stages of the illness, and then showed peripheral pulmonary artery dilation 
and diffused GGO changes in both lungs as the disease progresses.

CT pulmonary angiography of a vast majority of PTTM patients showed a widened main pulmonary artery and an enlarged right heart, indicating pulmonary hypertension, with no clear signs of pulmonary embolism $(11,12,14)$. The above findings may be explained by the fact that PTTM lesions are located in small arteries, while pulmonary angiography is effective only for blood vessels above $2 \mathrm{~mm}$. As shown by Toyonaga et al. dynamic observations of PTTM pulmonary angiography, there were no signs of pulmonary embolism at both the early stage and in the reexamination as the disease progressed (16).

ECG examination of most PTTM patients showed pulmonary hypertension, right ventricular enlargement, normal left ventricular ejection fraction, and decreased right ventricular ejection fraction $(10,11,13)$. Nearly half of PTTM patients had an enlarged right heart (7). Vincent et al. (17) reported that a PTTM patient whose ECG showed normal right ventricular systolic pressure in the initial stage had significantly increased two months later. Their right ventricle was moderately enlarged with good right ventricular systolic function, no right ventricular hypertrophy, and no left atrial enlargement.

CT pulmonary angiography was not tolerated in our patient due to his critical condition. The typical imaging features of PTTM revealed by chest CT examination provided us with important evidence for diagnosis.

\section{Pathological molecular mechanism}

The pathogenesis of PTTM is still unclear. Currently, the accepted pathogenesis is that malignant tumor cells form tiny tumor emboli in pulmonary blood vessels, which destroy vascular endothelium, form a pre-coagulation environment, and then activate the coagulation system and diffusely embolize the microvessels. This in turn leads to thrombocytopenia and intimal fibrocyte hyperplasia in small pulmonary blood vessels, lumen stenosis, pulmonary vascular structure remodeling, and ultimately to pulmonary hypertension (1). Tumor cells express a large number of tissue factors (TF) and VEGF, which play an important role in tumor metastasis, angiogenesis, growth, and thrombosis, and can induce vascular endothelial damage and further activate the coagulation system (18). The injured vascular endothelium secretes PDGF, osteopontin (OPN), and a variety of vasoactive factors. OPN can promote the adhesion, migration, and proliferation of fibroblasts, vascular endothelial cells, and smooth muscle cells. Cluster of differentiation 44 (CD44) is a cell adhesion molecule that can induce inflammation, autoimmune diseases, angiogenesis, and atherosclerosis. Tumor thrombus immunohistochemistry has shown that the positive rate of VEGF and TF were $96.6 \%$ and $100 \%$, respectively (8). Therefore, the detection of the expression of various molecules in tumor cells of PTTM patients may provide a basis for targeted biological therapy (5).

\section{Diagnosis and differential diagnosis}

Currently, there are no clear internationally recognized diagnostic criteria for PTTM, and the clinical manifestations of PTTM lack specificity. Pathological biopsy is the benchmark for PTTM diagnosis (18). However, due to the typically severe condition and the rapid progression of the disease at the time of admission, it is difficult for PTTM patients to tolerate invasive biopsy. The pathogenesis of PTTM is malignant tumors. Carter et al. (11) considered PTTM to be a kind of paraneoplastic syndrome. Some researchers have proposed that positive serum VEGF and D-dimer elevation should be regarded as the early diagnostic criteria for PTTM (19). Typically, patients with progressive dyspnea accompanied by hypercoagulable blood will not exhibit signs of pulmonary embolism on CT pulmonary angiography. For patients presenting with unexplained pulmonary hypertension, especially those with adenocarcinoma, clinicians should seriously consider PTTM. If pathology is not available, other clinical features, such as D-dimer and FDP elevation, pulmonary hypertension, chest HRCT, and PET-CT signs should be taken into consideration to assist clinical diagnosis.

The main diseases to be differentiated from PTTM are pulmonary embolism, PLC, and pulmonary veno-occlusive disease (POVD). These diseases have similar clinical manifestations: (I) pulmonary embolism is a general term for a group of diseases that cause pulmonary circulatory disorders due to the obstruction of pulmonary arteries or their branches by various endogenous or exogenous emboli. The typical ECG manifestations of pulmonary embolism are SI, QIII, and TIII patterns. Pulmonary hypertension in pulmonary embolism is largely caused by the mechanical occlusion of pulmonary arteries by emboli. For a pulmonary embolism, CT angiography of the pulmonary artery often shows embolus obstruction, which is generally opposite to PTTM CT angiography. (II) PLC is an interstitial lung change in which tumor cells spread along the lung 
lymphatic vessels and grow diffusely in lymphatic vessels, which represents a rare type of lung metastasis. PLC chest CT shows small nodule-like shadows spreading along the bronchial blood vessels bundle and/or lobular septum under the pleura, as well as increased and irregularly thickened lung markings. There is nodular thickening of the interlobular septum, while the lobular structure is normal. Only a small number of PLC patients develop pulmonary hypertension, which is accompanied by PTTM in some cases. (III) POVD is caused by the involvement of the posterior vascular system of pulmonary capillaries. Chest HRCT shows thickening of the peripheral lobular septum and ground glass nodule shadows (mainly in the central lobule). POVD and PTTM are primarily differentiated by their respective pathological characteristics. POVD is mainly caused by the extensive filling and occlusion of pulmonary veins by fibrous tissue, while PTTM is caused by small tumor emboli in veins and embolus-induced endovascular fibrosis. The case of this patient is accompanied by PLC, but pulmonary embolism and POVD are consistent with the characteristics of this case.

Currently, there are three main challenges in diagnosing PTTM. (I) The clinical manifestations, such as dyspnea, are not specific, and even no known lung cancer. (II) The clinical condition rapidly deteriorates, often with little time for diagnosis and treatment, and death is almost inevitable (12). (III) The patients' condition is generally critical, which increases the difficulty and risks of examination. Gorospe Sarasúa et al. (14) suggested that PTTM should be diagnosed in patients with malignant tumors that are accompanied by severe dyspnea and/or the development into acute or subacute pulmonary heart disease, especially when the chest CT shows a "tree-in-bud pattern", despite there being no features of respiratory system infection and no pulmonary emboli discovered by CT pulmonary angiography. Moon et al. (12) holds the views that PTTM patients are generally in a critical condition and cannot tolerate invasive examination, which is coupled with a rapid disease progression. Therefore, pathological diagnosis wastes time, and a biopsy is unnecessary for pathological diagnosis. Katayama et al. (10) reported that it is extremely difficult to make pathological diagnoses in PTTM patients prior to death, and clinical diagnosis is crucial for improving prognosis. PTTM is suspected if there are a large number of clinical indicators (including potential tumors and pulmonary hypertension) and can be clinically diagnosed after the exclusion of pulmonary hypertension caused by other factors (such as valvular heart disease and COPD). In addition, microangiopathic hemolytic anemia and DIC are also helpful for PTTM diagnosis. Endicott-Yazdani et al. (20) suggested that serum VEGF and D-dimer levels could be used as screening tools for suspected PTTM and disease condition evaluation.

In this study, the patient had a history of lung adenocarcinoma. He was clinically diagnosed with PTTM based on the typical clinical symptoms (such as dyspnea), multiple clinical indicators (such as elevated D-dimer and FDP), DIC, right heart failure, typical chest CT signs, and abnormally elevated LDH.

\section{Treatment progress}

At present, PTTM lacks effective treatment and has a poor prognosis, with a mortality rate of more than $90 \%$. The median survival time, from the time that the patient requires oxygen support, is 9 days (5). For a long time, multiple methods have failed to achieve a sufficient curative effect. Timely and effective comprehensive oncotherapy could clear tumor cells from the pulmonary circulation, which could eliminate the stimulation of vascular endothelial cells and alleviate pathophysiological processes $(18,21)$.

\section{Anti-tumor therapy}

PTTM is a fatal complication caused by tumor lung metastases. Effective oncotherapy for the primary disease is key to rescue. Considering the numerous clinical attempts over the years, now a day there is meta-analysis or case report, no systemic clinical research report data, lacking of effective treatment with extremely poor prognosis. Takahashi et al. (22) reported that one human epidermal growth factor receptor 2 (HER2)-positive PTTM patient survived up to 2 years after treatment with trastuzumab. Moon et al. (12) reported two cases of PTTM patients whose survival time was not prolonged even though the primary tumor was actively treated. Wakabayashi et al. (23) proposed that the expression of various molecules in tumor cells should be detected, as they can provide greater evidence for clarifying the pathogenesis of PTTM and targeted drug therapy. Kayatan et al. (15) showed that a combination treatment involving chemotherapy coupled with the anti-VEGF receptor antibody, bevacizumab $(8,15)$, anticoagulant therapy, and drugs for lowering pulmonary hypertension [such as endothelin antagonists (sildenafil), cyclic prostaglandin analogs, and phosphodiesterase-5 inhibitors (bosentan)] is effective in some cases of PTTM. However, anticoagulation therapy and lowering pulmonary hypertension remain controversial (24,25). Higo et al. (8) 
reported a case of colorectal cancer complicated with PTTM; in this case, symptoms such as dyspnea and dry cough were significantly improved following treatment with imatinib, bevacizumab, tigio, and cisplatin. Also, serum levels of VEGF, PDGF, and pro-BNP were decreased, with an approximate survival time of 1-year, which suggested that combined treatment with chemotherapy and targeted therapy alleviates PTTM progression.

In our case, when the patient developed rapidly progressive dyspnea and cough, PTTM was highly active. PTTM was diagnosed clinically after general discussion and consultation, recognition of the combined clinical manifestations, including increased D-dimer and FDP, anemia with abnormally elevated $\mathrm{LDH}$, right ventricular dysfunction, hypoxemia, and typical chest CT features. Therapeutically, considering the critical nature of PTTM and the accompanying serious complications (i.e., myocardial damage and severe thrombocytopenia), as well as the potentially severe adverse reactions of anti-tumor drugs, it is extremely difficult to treat PTTM clinically, despite the potential alleviating effects of treatment.

By means of comprehensive care, and taking into consideration the high VEGF expression in PTTM tumor cells, we arranged for treatment with the VEGF monoclonal antibody, bevacizumab, combined with pemetrexed oncotherapy, as well as comprehensive treatments involving anticoagulants (low molecular weight heparin sodium), transfusion of platelets and plasma, drugs for lowering pulmonary hypertension (vasodilation), correction of right ventricular insufficiency, oxygen therapy, treatment of lung infections, reliever medications, and nutritional support. The patient's condition was rapidly relieved; dyspnea was significantly alleviated, blood oxygen elevated, chest CT findings improved, DIC index returned to normal, edema disappeared, platelets increased, and the physical performance status was also substantially improved. This remarkable remission of illness also preliminarily verified the clinical diagnosis and treatment of PTTM, and improved clinical awareness of diagnosis and treatment of the disease.

\section{Anti-angiogenesis}

The use of the anti-angiogenic drug, bevacizumab, is worthy of serious consideration. The clinical pathophysiological changes of PTTM are mainly due to: (I) malignant carcinoma progression, tumor cell dissemination to the pulmonary blood and lymphatic vessels, and infiltration into the surrounding lumen (1,15); (II) the binding of the highly expressed VEGF molecule on the cell surface to the VEGF on vascular endothelial cells stimulates intima proliferation and thickening, resulting in intimal proliferation and lumen stenosis $(1,26)$; (III) tumor cell emboli activation of the coagulation system, leading to thromboembolism of microvessels, which is not only limited to the lungs, but also occurs in the micro-vessels of other organs, ultimately leading to multiple organ functional damage and even failure $(1,3)$. These pathophysiological processes are all associated with angiogenesis-related factors. Studies have reported that VEGF, PDGF, TF, and OPN serum levels are increased significantly in PTTM patients, and thus, can be used as biological indicators for PTTM diagnosis and prediction, as well as for the evaluation of therapeutic effects (2).

Anti-angiogenesis drugs block the combination of tumor cells with vascular endothelial cells, inhibit the proliferation stimulation response to vascular endothelial cells, modify the pulmonary vascular structure, and rapidly improve the lung tumor microenvironment. There have been reports showing that PTTM patients are relieved after bevacizumab therapy, and therefore, it may be a potentially effective drug for the treatment of PTTM. In addition, the normalization of tumor blood vessels by anti-angiogenic drugs enhances the antitumor effects of chemotherapy drugs, and the combination of these drugs has shown synergistic effectiveness.

It is generally considered that DIC and thrombi are contraindications to angiogenesis inhibitors. Thromboembolic events and thrombocytopenia are common side effects of bevacizumab. Serum VEGF of tumor patients is mainly derived from platelets, and $97 \%$ of bevacizumab binds to serum VEGF $(27,28)$. It has been demonstrated that platelets can absorb bevacizumab and release it at the site of the endothelial injury to participate blood coagulation and intimal proliferation. These results indicate that the target of bevacizumab is closely related to coagulation and thrombosis, although the complex interactions between them require further investigation.

\section{Anticoagulation therapy}

It is well established that patients with malignant tumors exhibit a hypercoagulable state, and anticoagulation therapy is recommended for these patients. Bevacizumab combined with anticoagulation therapy can significantly reduce the risk of thrombosis for patients with high coagulation risk (28). The patient in our study developed severe coagulation abnormalities, accompanied by DIC and severe thrombocytopenia. Although the reduction of platelets was related to bone marrow invasion, the platelets were remarkably increased after the DIC was relieved, indicating the influence of DIC on platelet consumption and 
thrombocytopenia. Furthermore, although the combination of anticoagulation and VEGF inhibitors will increase the risk of bleeding, the administration of low molecular weight heparin and fresh-frozen plasma in a reasonable dose and order to establish a balance between bleeding and coagulation is conducive to the correction of DIC.

\section{Anti-inflammation and other treatments}

There is also a report showing that steroid pulse therapy might be effective for the treatment of PTTM (29). However, further reports focusing on PTTM cases found that steroid treatment did not improve the condition (7,30,31). Gainza et al. (7) reported that plasma replacement treatment was ineffective or even counterproductive in PTTM patients.

\section{Conclusions}

PTTM is a rapidly developing and fatal tumor-associated syndrome with an extremely difficult pathological diagnosis. With an increasing number of patients, we will learn more about its pathogenesis and clinical pathophysiological changes. However, there is still a lack of diagnostic criteria and effective treatment methods, and the prognosis is extremely poor. Awareness and recognition of the disease, early identification of some clinical indicators, and practical auxiliary examinations are crucial to the early diagnosis of PTTM. Also, timely and effective anti-tumor treatment could relieve symptoms and improve prognosis. The significantly-increased serum VEGF, PDGF, TF, and OPN levels can be used as biological indicators for PTTM diagnosis and prediction, and for therapeutic effect evaluation. Bevacizumab may be a potentially effective drug for the treatment of PTTM; however, more clinical observation and basic research are required.

\section{Acknowledgments}

Funding: None.

\section{Footnote}

Reporting Checklist: The authors have completed the CARE reporting checklist. Available at http://dx.doi.org/10.21037/ apm-20-2220

Conflicts of Interest: All authors have completed the ICMJE uniform disclosure form (available at http://dx.doi. org/10.21037/apm-20-2220). The authors have no conflicts of interest to declare.

Ethical Statement: The authors are accountable for all aspects of the work in ensuring that questions related to the accuracy or integrity of any part of the work are appropriately investigated and resolved. All procedures performed in studies involving human participants were in accordance with the ethical standards of the institutional and/or national research committee(s) and with the Helsinki Declaration (as revised in 2013). Written informed consent was obtained from the patient.

Open Access Statement: This is an Open Access article distributed in accordance with the Creative Commons Attribution-NonCommercial-NoDerivs 4.0 International License (CC BY-NC-ND 4.0), which permits the noncommercial replication and distribution of the article with the strict proviso that no changes or edits are made and the original work is properly cited (including links to both the formal publication through the relevant DOI and the license). See: https://creativecommons.org/licenses/by-nc-nd/4.0/.

\section{References}

1. von Herbay A, Illes A, Waldherr R, et al. Pulmonary tumor thrombotic microangiopathy with pulmonary hypertension. Cancer 1990;66:587-92.

2. Godbole RH, Saggar R, Kamangar N. Pulmonary tumor thrombotic microangiopathy: a systematic review. Pulm Circ 2019;9:2045894019851000.

3. Thrombosis, Hemostasis Group HSoCMA. Consensus of Chinese experts on diagnosis of disseminated intravascular coagulation (version 2017). Zhonghua Xue Ye Xue Za Zhi 2017;38:361-3.

4. Papageorgiou C, Jourdi G, Adjambri E, et al. Disseminated Intravascular Coagulation: An Update on Pathogenesis, Diagnosis, and Therapeutic Strategies. Clin Appl Thromb Hemost 2018;24:8S-28S.

5. Uruga H, Fujii T, Kurosaki A, et al. Pulmonary tumor thrombotic microangiopathy: a clinical analysis of 30 autopsy cases. Intern Med 2013;52:1317-23.

6. Chinen K, Tokuda Y, Fujiwara M, et al. Pulmonary tumor thrombotic microangiopathy in patients with gastric carcinoma: an analysis of 6 autopsy cases and review of the literature. Pathol Res Pract 2010;206:682-9.

7. Gainza E, Fernandez S, Martinez D, et al. Pulmonary tumor thrombotic microangiopathy: report of 3 cases and review of the literature. Medicine (Baltimore) 2014;93:359-63. 
8. Higo K, Kubota K, Takeda A, et al. Successful antemortem diagnosis and treatment of pulmonary tumor thrombotic microangiopathy. Intern Med 2014;53:2595-9.

9. Yang BY. Climical recognition and management of thrombocytopenia in solid tumor. Zhonghua Yi Xue Za Zhi 2019;99:561-5.

10. Katayama D, Kuriyama K, Kinoshita T, et al. Pulmonary tumor thrombotic microangiopathy caused by prostate carcinoma. Acta Radiol Open 2016;5:2058460116662300.

11. Carter CA, Browning R, Oronsky BT, et al. The Case of a Zebra That Was Misdiagnosed as a Horse: Pulmonary Tumor Thrombotic Microangiopathy, a New Paraneoplastic Syndrome, Mimicking PD-1-Induced Pneumonitis. Case Rep Oncol 2016;9:68-75.

12. Moon SY, Lee KH, Lee JS, et al. Acute cor pulmonale due to pulmonary tumor thrombotic microangiopathy in two patients with breast cancer. Korean J Intern Med 2017;32:190-4.

13. Ho AL, Szulakowski P, Mohamid WH. The diagnostic challenge of pulmonary tumour thrombotic microangiopathy as a presentation for metastatic gastric cancer: a case report and review of the literature. BMC Cancer 2015;15:450.

14. Gorospe Sarasúa L, Ureña-Vacas A, García-Santana E. Radiological diagnosis of pulmonary tumor thrombotic microangiopathy: A non-bronchial cause of «tree-in-bud» pattern on computed tomography. Arch Bronconeumol 2016;52:621-2.

15. Kayatani H, Matsuo K, Ueda Y, et al. Pulmonary tumor thrombotic microangiopathy diagnosed antemortem and treated with combination chemotherapy. Intern Med 2012;51:2767-70.

16. Toyonaga H, Tsuchiya M, Sakaguchi C, et al. Pulmonary Tumor Thrombotic Microangiopathy Caused by a Parotid Tumor: Early Antemortem Diagnosis and Long-term Survival. Intern Med 2017;56:67-71.

17. Vincent F, Lamblin N, Classe M, et al. Subacute right heart failure revealing three simultaneous causes of post-embolic pulmonary hypertension in metastatic dissemination of breast cancer. ESC Heart Fail 2017;4:75-7.

18. Godbole R, Saggar R, Zider A, et al. Insights on pulmonary tumor thrombotic microangiopathy: a seven-patient case series. Pulm Circ 2017;7:813-20.

19. Kuwabara H, Yoshida S, Takasu T, et al. Pulmonary tumor thrombotic microangiopathy caused by gastric cancer. Ann Thorac Med 2012;7:168-9.

20. Endicott-Yazdani T, Ghazi A, Armstrong D, et al. Fatal pulmonary tumor thrombotic microangiopathy caused by undiagnosed metastatic gastric adenocarcinoma. Proc (Bayl Univ Med Cent) 2015;28:482-3.

21. Nayyar D, Muthiah K, Hayward CS, et al. Pulmonary tumor thrombotic microangiopathy from metastatic prostate carcinoma. Case Rep Pulmonol 2015;2015:286962.

22. Takahashi Y, Uruga H, Fujii T, et al. Antemortem diagnosis of pulmonary tumor thrombotic microangiopathy in a patient with recurrent breast cancer: a case report. BMC Cancer 2016;16:666.

23. Wakabayashi Y, Iwaya M, Akita M, et al. Pulmonary Tumor Thrombotic Microangiopathy Caused by Urothelial Carcinoma Expressing Vascular Endothelial Growth Factor, Platelet-derived Growth Factor, and Osteopontin. Intern Med 2016;5 5:651-6.

24. Kumar N, Price LC, Montero MA, et al. Pulmonary tumour thrombotic microangiopathy: unclassifiable pulmonary hypertension? Eur Respir J 2015;46:1214-7.

25. Minatsuki S, Miura I, Yao A, et al. Platelet-derived growth factor receptor-tyrosine kinase inhibitor, imatinib, is effective for treating pulmonary hypertension induced by pulmonary tumor thrombotic microangiopathy. Int Heart J 2015;56:245-8.

26. Price LC, Wells AU, Wort SJ. Pulmonary tumour thrombotic microangiopathy. Curr Opin Pulm Med 2016;22:421-8.

27. Verheul HM, Lolkema MP, Qian DZ, et al. Platelets take up the monoclonal antibody bevacizumab. Clin Cancer Res 2007;13:5341-7.

28. Kazazi-Hyseni F, Beijnen JH, Schellens JH. Bevacizumab. Oncologist 2010;15:819-25.

29. Miyazaki S, Ikeda T, Ito G, et al. Pulmonary tumor thrombotic microangiopathy successfully treated with corticosteroids: a case report. J Med Case Rep 2017;11:356.

30. Higashi A, Dohi Y, Uraoka N, et al. The Potential Role of Inflammation Associated with Interaction between Osteopontin and CD44 in a Case of Pulmonary Tumor Thrombotic Microangiopathy Caused by Breast Cancer. Intern Med 2015;54:2877-80.

31. McAnearney S, Drain M. A case of pulmonary tumour thrombotic microangiopathy. Respir Med Case Rep 2015;16:7-10.

Cite this article as: Lu L, Wang Z, Li H, Li X, Ma S, Wang L, Yang B. Bevacizumab combined with pemetrexed successfully treated lung adenocarcinoma complicated with pulmonary tumor thrombotic microangiopathy: a case report and literature review. Ann Palliat Med 2021;10(1):767-777. doi: 10.21037/apm20-2220 